ULRICH ENGEL

Termini für Deutsch als Fremdsprache

- Praxis, Mängel, Möglichkeiten -

Inhal tsübersicht

1. Zur Rolle der Grammatik im Fremdsprachenunterricht

2. Zur Terminologie im Bereich Deutsch als Fremdsprache

3. Geprüfte Materialien

4. Zur Rezeption grammatischer Terminologie in Lehrwerken für Deutsch als Fremdsprache

5. Festgestellte Mängel

6. Konkrete Vorschläge

Literaturverzeichnis

1. Zur Rolle der Grammatik im Fremdsprachenunterricht

Es ist oft gesagt worden, aber es kann offenbar nicht oft genug wiederholt werden, das die Funktion der Grammatik im muttersprachlichen Unterricht und im Fremdsprachenunterricht grundsätzlich verschieden ist. Uber die folgenden Grundsätze ist sich die Fachwelt weitgehend einig:

- Im muttersprachlichen Unterricht ist die Grammatik weitgehend Selbstzweck. Das heibt: sie wird in erster Linie behandelt, damit die Lernenden sie kennenlernen, verstehen, mit ihr umgehen können usw.

2war wird oft behauptet, der muttersprachliche Grammatikunterricht diene der Ausbildung und Förderung der sprachlichen Kompetenz. Aber eine Nachprüfung sämtlicher Lehrwerke ergibt, das die kommunikative Kompetenz zwar auf vielfältige Weise entwickelt werden kann, dab der Grammatikunterricht dazu aber nichts oder doch nur verschwindend wenig beiträgt.

Einige einsichtige (oder ehrliche) Lehrbuchautoren weisen indessen darauf hin, daß der muttersprachliche Grammatikunterricht u.a. auch Einblicke in die struktur der Muttersprache eröffnen könne. Dies ist meines Erachtens seine wesentliche Funktion. 
- Im Fremdsprachenunterricht hingegen hat die Beschäftigung mit der Grammatik eine völlig untergeordnete Funktion. Hauptziel des Fremdsprachenunterrichts ist der Erwerb der zielsprachlichen Kompetenz. Beschäftigung mit der Grammatik ist in diesem zusammenhang nur legitim, sofern sie zum Kompetenzerwerb und zur Kompetenzerweiterung beiträgt. Der alte Grundsatz

So wenig Grammatik wie möglich,

so viel Grammatik wie nötig

wird durch die Unterrichtspraxis immer wieder aufs neue bestätigt. Dies bedeutet aber auch: Der Fremdsprachenunterricht soll zwar nicht grammatikgesteuert sein, aber er mus in jedem Falle grammatikf u $n$ d $i$ e $t$ sein.

Fremdsprachenwerke müssen daher auf einer bestimmten Grammatikkonzeption beruhen. Damit soll nicht gesagt sein, daB ein Modell alle Teile eines Lehrbuchs durchgehend bestimmen müsse. Eine theoretische Grundlegung aus verschiedenen Grammatikmodellen ist durchaus denkbar und wurde auch nicht ohne Erfolg praktiziert. Es bleibt aber die Forderung, daß ein Unterrichtswerk ein erkennbares grammatisches Fundament haben muB; und es bleibt die weitere Forderung, daß dieses grammatische Fundament in einer das Lehrwerk begleitenden Grammatik vorliegen muB: Der Lehrer (und durchaus auch der fortgeschrittene Schüler) muß in zweifelsfällen nachschlagen können.

Im folgenden wird davon ausgegangen, daß Grammatik die struktur der zu erlernenden sprache in irgend angemessener Weise wiedergibt. Und es wird die Voraussetzung gemacht, daß der Erwerb dieser sprache ohne Kenntnis ihrer struktur nicht möglich ist. Ich bin mir durchaus der altersbedingten Variationen der Sprachlernmethoden bewußt. Es soll hier auch keineswegs einer Wiederholung des alten grammatikalisierenden Fremdsprachenunterrichts das wort geredet werden. Aber die Lernprogression, vor allem in ihrem Ubungsteil, muB auf einer fabbaren und mindestens dem Lehrer zugänglichen Grammatik aufgebaut sein. Aus diesem Grunde ist Fremdsprachenunterricht ohne Grammatik nicht einmal als schlagwort ernst $z u$ nehmen. Das Lehrwerk Deutsch 2000 zum Beispiel, das zunächst ohne grammatische Kom- 
ponente eingeführt wurde - freilich in der erklärten Absicht, auf diese Weise dem Lehrer die Freiheit der Wahl für ein beliebiges Grammatikmodell zu überlassen -, muBte sehr bald eine begleitende "Grammatik der modernen deutschen Umgangssprache" nachschieben, die allerdings durchaus nicht alles bietet, was man heute von einer Grammatik erwarten darf.

Jede Grammatik braucht Termini, und da ihre wirkung auch von der Terminologie abhängt, ist das Benennungsproblem im Fremdsprachenunterricht besonders wichtig.

2. Zur Terminologie im Bereich Deutsch als Fremdsprache

Nach dem Gesagten muB, wer sich im Fremasprachenunterricht engagiert, über die verwendete Terminologie nachdenken. Das kann in den Lehrbüchern oder auch nur in den Lehrer-Handbüchern geschehen; es reicht aber nicht, wenn es nur im kopf des Lehrbuchautors oder des Lehrers geschieht.

Da ergeben sich Fragen der folgenden Art:

1. Welche grammatische Konzeption liegt dem Lehrbuch zugrunde? Dies ist zwar nicht im engeren sinn Thema unserer Betrachtung. Aber die Frage nach der grammatischen Konzeption taucht allenthalben auf, sie wird von Lektion $\mathrm{zu}$ Lektion unvermeidlicher. Denn ohne die Einführung bestimmter Phrasen und ihrer strukturen, ohne die Auseinandersetzung mit fakultativen objekten (Ergänzungen), ohne die Bekanntmachung mit bestimmten Kategorien der Wortstellung, der satzklammer etwa, ohne wenigstens klare vorstellungen über die Relation von Tiefenkasus und oberflächenkasus kann kein Unterricht betrieben werden. Vielleicht war das immer so: unsere Epoche ist dadurch gekennzeichnet, das solche probleme zum Teil erst formuliert wurden, daß man sie nicht mehr ignorieren kann.

2. Welche Termini werden aus der grundlegenden Grammatikkonzeption übernommen, welche werden adaptiert, und wie werden sie adaptiert? 
3. Inwiefern sind diese Termini geeignet für den Fremasprachenunterricht?

Diesen Fragen will ich nachgehen anhand neuerer Lehrwerke für Deutsch als Fremdsprache, konkret: Lehrwerken, die in Deutschland seit $1977 / 78$ neu erschienen sind. Zum Vergleich werde ich in Polen hergestellte und verwendete Deutsch-Lehrbücher heranziehen und dabei, da sich der Markt dort nicht so schnell verändert, auch etwas weiter zurückgreifen.

\section{Geprüfte Materialien}

Die in Deutschland erschienenen Lehrwerke wenden sich prinzipiell an Lernende mit beliebiger Muttersprache. zwar gibt es für manche Lehrwerke auch überarbeitete zweisprachige Versionen; sie spielen jedoch auf dem Lehrmittelmarkt eine Nebenrolle. Ich habe mich bemüht, alle derartigen Lehrwerke zu erfassen, die mir bis Sommer 1984 zugänglich waren.

Den kurzen bibliographischen Angaben folgt im allgemeinen eine knappe charakterisierung, vor allem hinsichtlich der zielgruppe.

1. Häussermann et al.: SPRACHKURS DEUTSCH, 1978, 4 Teile. Ein Lehrwerk für Intellektuelle aller Altersstufen.

2. Neuner et al.: DEUTSCH AKTIV, 1979f., 2 Teile.

Ein Lehrwerk für Erwachsene, mit vorwiegend pragmatischkommunikativer Funktion, wobei jedoch kognitives Vorgehen bewuBt einbezogen wird.

DEUTSCH HIER, 1982 .

Eine Variante von DEUTSCH AKTIV für ausländische Arbeitnehmer; der Anteil der Grammatik tritt hier deutlich zurück.

DEUTSCH KONKRET, 1983.

Eine Variante von DEUTSCH AKTIV für ausländische Jugendliche; der Grammatikanteil ist hier noch geringer. 
3. Aufderstraße et al.: THEMEN, 1983.

Ein Lehrbuch für Lernende ab 16 Jahre, das auf einer kommunikativen konzeption beruht.

Die Grammatik ist in "grammatischen Ubersichten" des Kursbuchs niedergelegt.

4. Hieber: LERNZIEL DEUTSCH, 1983.

Ein Lehrbuch für Lernende ab 16 Jahre, die kognitiv lernen wollen. Die Grammatik findet sich großenteils im Lehrerhandbuch thematisiert.

Die bisher genannten Lehrwerke waren im wesentlichen einer grammatischen Konzeption verpflichtet, der sogenannten DependenzVerb-Grammatik (DVG). 1 sie sind dabei weitgehend orientiert am ZERTIFIKAT DEUTSCH ALS FREMDSPRACHE, ${ }^{2} 1977\left(={ }^{3} 1985\right)$.

herausgegeben vom deutschen volkshochschulverband und vom Goethe-Institut.

Dieses zertifikat enthält die prüfungsanforderungen für die Grundstufe im Fach Deutsch als Fremdsprache.

KONTAKTSCHWELLE DEUTSCH ALS FREMDSPRACHE, 1981.

Eine zusammenstellung von Prüfungsanforderungen, die deutlich stärker kommunikativ orientiert ist als das zertifikat, aber ein darunter liegendes Niveau anstrebt.

Sowohl das zertifikat wie die Kontaktschwelle sind in ihrem syntaktischen Teil an der DVG orientiert.

Die folgenden in der Bundesrepublik erstellten Lehrwerke für Deutsch als Fremdsprache orientieren sich an anderen Grammatikmodellen:

5. Becker et al.: DIALOG DEUTSCH, 1978.

Es handelt sich um einen kommunikativen Sprachkurs der Carl Duisberg Zentren, der in erster Linie für Fach- und Führungskräfte der wirtschaft bestimmt ist. Grammatische Terminologie findet sich hier nur im Lehrerhandbuch. 
6. Bieler: MITEINANDER, 4 Bände, $1980 \mathrm{ff}$.

Ein Lehrbuch für die deutschen Auslandsschulen. Alle grammatischen Informationen finden sich im Text- und Arbeitsbuch. Zuständig für die Grammatik ist in diesem Lehrwerk der versierte Linguist und Didaktiker Gerhard Kaufmann.

7. Schäpers et al.: GRUNDKURS DEUTSCH, 1980.

Für Lernende ab 16 Jahre. Grammatische Terminologie findet sich hier nur im Lehrerhandbuch. Dazu gibt es als Fortsetzung

Stalb: AUFBAURURS DEUTSCH, 1983.

Hier finden sich in jeder Lektion die Teile Texte - Redemittel - Grammatik.

8. Griesbach: DEUTSCH MIT ERFOLG.

Ein audiovisuelles Lehrwerk für Erwachsene. Es enthält einen grammatischen Uberblick auf 10 seiten.

Die zum Vergleich beigezogenen in Polen erstellten und verwendeten Lehrbücher sollen vor allem zeigen, wie auf kontrastivem Ansatz beruhende Lehrwerke sich heute darstellen. Es schien gerechtfertigt, polnische Deutschlehrwerke beizuziehen, da der Deutschunterricht in Polen immer noch eine wichtige Rolle spielt; auBerdem war es mir infolge persönlicher Beziehungen möglich, mir die wichtigsten derzeit in Gebrauch befindlichen Deutschlehrwerke in Polen $z u$ beschaffen.

An polnischen Mittelschulen, die im ungefähren unserer Sekundarstufe I der Gymnasien entsprechen, sind vor allem folgende Lehrwerke im Gebrauch:

9. Honsza/Kozielek: LERNT MIT UNS! 4 Bände, 1976. Jede Lektion enthält einen Grammatikteil.

10. Honsza/Koczy: ANFANG UND FORTSCHRITT, Band 1, 1983. Dieses neue Lehrwerk soll das zuvor genannte ersetzen. Auch hier enthält jede Lektion einen Grammatikteil. 
11. Grucza et al.: Band 1, DER ERSTE SCHRITT, 1977.

Band 2, UNSER ALLTAG, 1978.

Band 3, ES GIBT IMMER PROBLEME, 1978.

In diesem Lehrwerk enthält jede Lektion einen Abschnitt

"Grammatik".

Für den Unterricht an polnischen Universitäten ist gedacht

12. Czochralski et al.: AKADEMICKI PODRECZNIK JĘZYKA NIEMIECKIEGO

(Akademisches Lehrbuch der deutschen Sprache), 1983.

In den 30 Lektionen sind viele Grammatikinformationen enthalten.

Das bisher meistgebrauchte Lehrwerk (das seine Vorrangstellung wahrscheinlich auch der Tatsache verdankt, daß es immer beziehbar war) ist

13. DEUTSCH. EIN LEHRBUCH FUR AUSLÄNDER.

Untersucht wurde Band 1, Leipzig ${ }^{16} 1976$.

4. Zur Rezeption grammatischer Terminologie in Lehrwerken für Deutsch als Fremdsprache

Die Tatsache, daß von den in der Bundesrepublik produzierten acht Lehrwerken vier auf der DVG beruhen bzw. sich auf sie berufen, bedingt eine weitgehende terminologische Einheitlichkeit. Es handelt sich dabei um SPRACHKURS DEUTSCH, DEUTSCH AKTIV, THEMEN und LERNZIEL DEUTSCH. Diese Lehrwerke haben, wenn auch mit gewissen Änderungen und Abstrichen, im wesentlichen die Terminologie der DVG übernommen. Der grammatisch besehen weitgehend traditionelle SPRACHKURS DEUTSCH verwendet Begriffe und Termini der DVG vor allem im satzbereich, kennt aber wie die ältere Grammatik nur insgesamt vier Ergänzungen. In DEUTSCH AKTIV findet man alle wesentlichen Ergänzungen der DVG (verständlicherweise nach dem stand von 1977), jedoch wird hier der Nominativergänzung eine sonderstellung vor allem bei den Modalverben 
eingeräumt. In THEMEN begegnet man den Ergänzungen der DVG, ebenfalls nach dem stand von 1977, in den "grammatischen Ubersichten ". Das Werk LERNZIEL DEUTSCH schlieblich, das weitgehend traditionelle Grammatik einführt, bringt Kategorien und Sehweisen der DVG vor allem im Bereich der Wortstellung.

Die übrigen in der Bundesrepublik produzierten Lehrwerke stützen sich im wesentlichen auf die traditionelle Grammatik der Nachkriegszeit.

Die polnischen Deutschlehrwerke DER ERSTE SCHRITT Usw., LERNT MIT UNS!, ANFANG UND FORTSCHRITT hingegen verwenden die traditionelle grammatische Terminologie, wie sie vor dem zweiten Weltkrieg üblich war.

Das von einem Kollektiv im Herder-Institut, Leipzig, erstellte Lehrwerk DEUTSCH. EIN LEHRBUCH FUR AUSLÄNDER. stützt sich auf die DEUTSCHE GRAMMATIK von Helbig/Buscha und verwendet im groben ganzen auch dessen Terminologie.

FaBt man dies alles zusammen, so muB man ein bedrückendes terminologisches Durcheinander feststellen. Vier westdeutsche Lehrwerke haben die DVG-Terminologie mehr oder minder unverändert rezipiert und kaum adaptiert: vier weitere westdeutsche Lehrwerke bleiben bei der neueren traditionellen Grammatik. Dies zeigt sich bei Definition und Benennung der Wortklassen, der objekte (man begegnet regelmäBig drei oder vier objekten) und der sonderstellung des subjekts.

In den vier geprüften polnischen Deutschlehrwerken herrscht die ältere Terminologie; dort liest man noch nichts vom Präpositionalobjekt, und die wortstellung wird nur sehr flüchtig und ganz im herkömmlichen sinne behandelt. Die Diskrepanzen werden vermehrt durch die Helbig/Buscha'sche Terminologie in DEUTSCH, die in wichtigen Bereichen eigene wege geht.

Gemeinsam ist den geprüften Lehrwerken lediglich, daB sie allesamt fast nur lateinische Terminologie verwenden; daß es dabei erhebliche Unterschiede gibt, ist bekannt. 
In SPRACHKURS DEUTSCH, DEUTSCH AKTIV, THEMEN und LERNZIEL DEUTSCH, die einer bestimmten Grammatikkonzeption verpflichtet sind, gibt es relativ wenige Unklarheiten und Widersprüche. Bedauerlich bleibt freilich, daß der SPRACHKURS DEUTSCH bei der alten "Konjunktion" bleibt, vor allem nachdem in der KONTAKTSCHWELLE so sorgsam zwischen Konjunktor und subjunktor unterschieden wird. Ärgerlich ist allemal, daß der SPRACHKURS DEUTSCH die Bezeichnung "Satzbauplan" für bestimmte stellungstypen verwendet und sich damit in widerspruch zur gesamten neuen Forschung setzt.

Nun kann die Tatsache, daß Konsistenz in der Terminologie durch strikte Anlehnung an ein wissenschaftliches Grammatikmodell herbeigeführt wurde, noch nicht als vorzug eines Lehrwerks gelten; hier mus zugleich gefragt werden, ob die Termini nicht zu esoterisch, zu umständlich oder einfach zu schlecht aussprechbar seien. In dieser Hinsicht sind die genannten vier Lehrwerke allerdings nicht in die pflicht zu nehmen, da sie im wesentlichen den vorgaben des 2ERTIFIKATS und der KONTAKTSCHWELLE folgen.

Was die übrigen Lehrwerke betrifft - und teilweise eben auch die DVG-basierten -, so müssen erhebliche terminologische Mängel konstatiert werden, vor allem bei der Benennung von Wortklassen, Flektionskategorien, wortteilen, Satzgliedern und Wortstellungserscheinungen. In dieser Reihenfolge werden die wesentlichen Mängel aufgelistet.

\subsection{Wortklassen}

Hier stört gelegentlich das Nebeneinander konkurrierender Bezeichnungen für dieselbe sache. So verwendet MITEINANDER undifferenziert die Bezeichnungen "Nomen" und "Substantiv". 
Fast allgemein versteht man unter "Pronomen" sowohl die autonom wie die attributiv gebrauchten Elemente, unterscheidet also terminologisch nicht zwischen mein und meiner usw. (MITEINANDER, GRUNDKURS DEUTSCH, LERNT MIT UNS, ANFANG UND FORTSCHRITT u.a.).

Von dem alten und grammatisch kaum motivierbaren Begriff der "Konjunktion", der Konjunktor und subjunktor zusammenfaßt, war schon die Rede; er findet sich weiter in DIALOG DEUTSCH, MITEINANDER, LERNT MIT UNS. Hier wurde eine wichtige Erkenntnis der neueren Linguistik zum Schaden der Lernenden einfach nicht akzeptiert.

Unausrottbar scheint offensichtlich die seltsame Bildung "Pronominaladverb" (AUFBAUKURS DEUTSCH, LERNT MIT UNS; CZOCHRALSKI u.a.). Dabei ist längst klargestellt, daB diese wörter - dafür, damit usw. - nichts von einem Pronomen an sich haben; kennzeichnend ist vielmehr, daB es sich um Partikeln, vielleicht Adverbien, handelt, die eine Präposition enthalten. Auch wenn man "Pronomen", "pronominal" lediglich durch eine Verweisfunktion definiert, ist der Ausdruck nicht zu rechtfertigen, weil auch die meisten Adverbien per se eine Verweisfunktion haben und der Ausdruck dann ein Pleonasmus wäre. So bietet sich vernünftigerweise der Terminus "Präpositionaladverb" an. In MITEINANDER wird die sache allerdings nicht besser, wenn dort "Präpositionaladverb" und "Pronominaladverb" als konkurrierende Bezeichnung für dieselbe sache verwendet werden.

\subsection{Flexion}

In einigen Lehrwerken stehen die Termini "Präteritum" und "Imperfekt" für dieselbe verbale Flexionskategorie nebeneinander (z.B. MITEINANDER). Das verwirrt, weil hinter der terminologischen Differenz begriffliche Unterschiede vermutet werden.

In vielen Lehrwerken begegnet man der seltsamen Kategorie des "Konditional", womit die anderwärts sogenannte würde-Umschreibung gemeint ist. In anderen Sprachen, wie dem Französischen oder (unter anderer Bezeichnung) dem serbokroatischen, existiert tatsächlich eine derartige Flexionskategorie; für das Deutsche 
sollte man sie umso weniger gelten lassen, als einfacher Konjunktiv und würde-Umschreibung in der Regel frei austauschbar oder morphonologisch bedingt sind. DaB in DEUTSCH MIT ERFOLG die würde-Umschreibung kurzerhand als normaler Konjunktiv II der schwachen verben aufgefabt wird, mag manchem etwas $z u$ pauschal klingen. Jedenfalls ist diese Auffassung aber wesentlich zutreffender als die Annahme einer eigenen Flexionskategorie, womöglich mit eigener Semantik. Durch die Beibehaltung dieses veralteten Terminus spiegelt z.B. MITEINANDER dem deutschlernenden Ausländer eine Schwierigkeit vor, die im Grunde nicht vorhanden ist.

\subsection{Wortbildung}

Der "Verbzusatz", der seit langer zeit praktisch ausschlieblich für die trennbaren Verbpräfixe verwendet wird, wird verschiedent1ich (z.B. MITEINANDER) für trennbare und untrennbare präfixe verwendet. Einer der Termini "Verbzusatz" und "Präfix" (des Verbs) wäre dann überflüssig; für den eigentlichen Verbzusatz müßte eine neue Bezeichnung gefunden werden.

DaB in LERNT MIT UNS und bei CZOCHRALSKI beständig von "trennbar zusammengesetzten Verben" gesprochen wird, scheint auf eine anderwärts verschollene Tradition zurückzugehen. Der Terminus ist einerseits irreführend, weil man gewöhnlich zwischen zusammensetzung (Komposition) und Ableitung unterscheidet und die fraglichen Verben (aufbrechen, mitnehmen) zu den abgeleiteten gerechnet werden; er ist andererseits, wenn man Ableitung als Sonderform der zusammensetzung auffabt, pleonastisch und darum unnötig.

\subsection{Der Satz}

Fast allgemein werden in den Lehrwerken die Satzglieder durch die "Verschiebeprobe" ermittelt. Es ist vielfach gezeigt worden ${ }^{2}$, daß diese probe nicht stichhaltig ist, weil es eine ganze Reihe von verschiebbaren stellungselementen gibt, die nicht als Satzglieder bezeichnet werden können. Zwar erfaBt die Verschie- 
beprobe zum größten Teil Satzglieder, und ihre Anwendung mag, vor allem im Anfängerunterricht, durchaus vertretbar sein. Es sollte aber, zumindest im Lehrerheft, ein deutlicher Vorbehalt gemacht werden; Lehrer und fortgeschrittene Lernende sollten auf jeden Fall wissen, daß die Verschiebeprobe kein volltaugliches Verfahren zur Ermittlung der Satzglieder ist.

In einer Reihe von Lehrwerken finden sich seltsame Doppelbezeichnungen für Satzglieder. Da werden die Termini "Ergänzung" und "Objekt" undifferenziert nebeneinander verwendet (LERNzIEL DEUTSCH), anderswo "Subjekt" und "Nominativergänzung", "Präpositionalobjekt" und "Präpositivergänzung". Und auch daß bestimmte Ergänzungen als "Angaben" bezeichnet werden, ist nicht gerade selten (z.B. LERNZIEL DEUTSCH).

Vom "Satzbauplan", der beim SPRACHKURS DEUTSCH für bestimmte Stellungstypen des Verbs verwendet wird, war schon die Rede; in der gesamten Forschung, die den Terminus überhaupt verwendet, meint er jedoch das Grundmuster eines satzes, der durch das Verb und die von ihm selegierten Ergänzungen gebildet wird.

\subsection{Wortstellung}

$\mathrm{Zu}$ diesem weiten und problemerfüllten Bereich ist nur anzumerken, daB die alte und bedenkliche Unterscheidung $z$ wischen "gerader" und "ungerader" Wortstellung in Honszas neuem Buch ANFANG UND FORTSCHRITT fröhliche Urständ feiert (hier allerdings als "gerade" vs. "versetzte" Wortfolge). Das Gefährliche ist die Sehweise, die hinter dieser Benennung steht: die Annahme näm$1 \mathrm{ich}$, daB das subjekt "normalerweise" im deutschen Hauptsatz an erster stelle stehe und dab die Voranstellung eines anderen Elements, die eine Hinanstellung des subjekts bedeutet, lediglich als "Umkehrung" dieser normalen Folge gewertet wird. Sätze wie Das weiB ich alles schon., Gestern war alles noch anders. und viele andere widersprechen dieser Annahme, weil sie eine Stellung aufweisen, die, wenn iberhaupt eine, als die normale bezeichnet werden mus. 
Insgesamt kann sich der Ärger über terminologische Mängel in neueren Deutschlehrwerken in Grenzen halten: Trotz der zu kritisierenden Erscheinungen wird in diesen Lehrwerken im allgemeinen verständich, was gemeint ist.

Weit mehr protest wäre anzumelden, wenn es um die grammatischen Begriffe geht, denen Regeln, Erläuterungen u.ä. zugrundeliegen. viele der unabdingbaren grammatischen Begriffe werden in den Lehrwerken überhaupt nicht eingeführt (vor allem im Bereich der Satzstruktur, der Wortstellung, der Textkonnexion), oder aber sie werden auf äußerst unzureichende, tolpatschige, irreführende Weise eingeführt.

Aber dies war nicht mein Thema. Ich hatte auszugehen von einer einmal gewählten (wenngleich oft disparaten) Grammatikkonzeption und zu fragen, wie sich diese Konzeption in Lehrwerken niederschlägt, wie sie didaktisiert wird.

Allgemein kann gesagt werden, daß Lehrwerke, die sich klar zu einem einheitlichen grammatischen Modell bekennen, besser wegkommen als andere, daß sie weniger Unklarheiten oder Widersprüche enthalten. Im übrigen reflektieren die beobachteten Mängel der Lehrwerke teilweise auch Defizite der Linguistik; und dies ist für mich - als Linguisten - ein besonderes Ärgernis. Termini wie "Konjunktion", "Konditional", "Pronominaladverb", "Satzglied" erregen nicht bloß deshalb Anstoß, weil sie unklar, uneinheitlich, widersprüchlich verwendet werden, auch nicht wegen der zu beanstandenden Begriffe, die sie bezeichnen, sondern vor allem deshalb, weil sie auch von vielen Linguisten noch in der skizzierten weise verwendet werden.

Es gibt gleichwohl Wege, terminologische schwierigkeiten zu verhindern oder mindestens zu verringern. Dazu müssen meines Erachtens sechs Forderungen erfüllt werden.

1. Man muB noch nachdrücklicher als bisher verlangen, das die Lehrbuchautoren ihre linguistischen Grundlagen offenlegen, auf vorhandene Referenzgrammatiken verweisen oder, falls 
keine existiert, selbst eine Referenzgrammatik mitliefern. Diese Forderung muß besonders gegenüber "traditionell" fundierten Lehrwerken erhoben werden. Ihre Erfüllung hätte den Vorteil, daß man in $\mathrm{Zwe}$ welsfällen nachschlagen kann.

2. Alle im Lehrbuch verwendeten Termini muissen an leicht zugänglicher stelle definiert werden. Diese Forderung ist besonders an die im Ausland erstellten Deutschlehrwerke $z u$ richten.

3. Man muB die Lernpraxis beobachten; man muB immer wieder fragen: Was wissen die Lernenden überhaupt? Was ist ihnen zumutbar? Was ist für sie notwendig?

4. Man muB den Lehrmittelmarkt beobachten. Wichtig ist auch, was angeboten und was verkauft wird, auch was "ankommt". Und es mus gefragt werden, ob sich dadurch unser grammatisches Grundwissen ändert.

5. Die Lehrer müssen in Fragen der Grammatik und der grammatischen Terminologie besser als bisher fortgebildet werden. Wer die Einführung neuerer Lehrwerke billigt, hat auch dafür Sorge zu tragen, daB man sie verwenden kann; videant ministeriales. Die Lehrer müssen in verschiedene Lehrwerke und verschiedene didaktische sehweisen eingeführt werden. Zu solchen Fortbildungsveranstaltungen sollte man Linguisten nur mit grobem Bedacht beiziehen, nicht zuletzt weil viele von ihnen allzusehr auf ihre eigenen sehweisen festgelegt sind.

6. In der Lehrerfortbildung müBten die Akzente richtig gesetzt werden: Es geht in allererster Linie um die VERMITTLUNG VON SPRACHE, um den Aufbau einer zielsprachlichen Kompetenz.

Gerade die letzte Forderung mus betont werden. Die neuerdings immer stärker erhobenen Forderungen nach einem verbesserten Deutschlandbild, nach Kulturrelativierung usw. mögen für sich genommen berechtigt sein. Aber sie setzen falsche akzente und drängen das Hauptproblem in den Hintergrund. Alle Kenntnis kultureller Besonderheiten und zusammenhänge hilft wenig, wenn die Lernenden nicht zum freien Reden gelangen, wenn sie sich nicht wenigstens auf Deutsch verständlich machen können. 
Für dieses zentrale Lernziel benötigt man Grammatik im Unterricht, wenn auch in wohl abzuwiegenden Dosen, und man benötigt dann auch Etiketten für die gewählten grammatischen Kategorien. Deshalb halte ich die Ubung, über grammatische Termini nachzudenken, auch weiterhin für kein anrüchiges Geschäft.

Für die DEUTSCH-SERBOKROATISCHE KONTRASTIVE GRAMMATIK ${ }^{3}$ des Instituts für deutsche Sprache, die 1986 erschien und sich an Lehrer, studenten und wissenschaftler wendet, war das Problem einer anwenderfreundlichen, aber wissenschaftlich stichhaltigen Terminologie ebenfalls zu lösen. Die wichtigsten dieser Termini sollen im folgenden, systematisch geordnet und bedarfsweise kurz erläutert, wiedergegeben werden. Dabei sind wir ${ }^{4}$ uns bewußt, das es sich um einen ersten Versuch handelt, der zweifellos korrekturbedürftig ist; für Anregungen sind wir daher besonders dankbar.

\subsection{Wortklassen}

Es werden in alphabetischer Reihenfolge die verwendeten wortklassen genannt und erforderlichenfalls kurze Erläuterungen hinzugefügt.

\section{ADVERB}

Hierunter fällt keineswegs alles, was in Grammatiken und Lehrwerken so genannt wird. Das Adverb ist definiert als unveränderliches Wort (Partikel), das jederzeit die erste Stelle im Hauptsatz einnehmen und als Antwort auf Sachfragen fungieren kann: damals, dort, deshalb u.a.

ADJEKTIV

Hierunter fallen nur die attributiv verwendbaren Adjektive, also neben blau, groB, alt auch ehemalig usw., nicht aber die "nur prädikativen Adjektive" der traditionellen Grammatik wie quitt, leid usw. (s. dazu "Sonstige Partikeln"). 
Da Adjektive gemäß ihrer Definition immer zwischen Determinativ und Nomen stehen können, sind auch die Kardinalzahlen Adjektive; es ist weder nötig noch gerechtfertigt, eine eigene Wortklasse "Numerale" anzusetzen.

\section{DETERMINATIV}

Hierunter fallen alle regelmäBigen "Begleiter" des Nomens (auBer den Adjektiven), also auBer den Artikeln auch die attributiv gebrauchten "Demonstrativpronomina", "Possessivpronomina" usw. der traditionellen Grammatik.

\section{KONJUNKTOR}

Hierher gehören alle nebenordnenden "Konjunktionen" der traditionellen Grammatik: und, aber, denn, oder u.a. Satzverbindende Konjunktoren lassen sich dadurch von satzverbindenden Adverbien unterscheiden, daß sie nicht die erste stelle im satz beanspruchen.

\section{NOMEN}

Alle Elemente dieser Wortklasse sind kasusvariabel und genuskonstant.

\section{PRÄPOSITION}

Es handelt sich um Partikeln, die jederzeit eine Nominalphrase in spezifischem (von der Präposition festgelegtem) Kasus bei sich haben können.

Damit fallen die partikeln als und wie nicht unter die präpositionen, weil sie keinen spezifischen Kasus verlangen.

\section{PRONOMEN}

Hierunter werden nur diejenigen flektierbaren wörter verstanden, die die einzige Funktion haben, Nominalphrasen zu ersetzen. Daher ist keiner ein pronomen, kein ein Determinativ. wörter wie dieser, die attributiv wie autonom auf gleiche weise flektiert werden, gehören ebenfalls zu den Determinativen. 
SONSTIGE PARTIKELN

Wir unterscheiden

ABTÖNUNGSPARTIKELN

Diese geschlossene Klasse ( $j a$, eben, schon usw.) ist weder erststellenfähig noch negierbar.

\section{GRADPARTIKELN}

Diese geschlossene Klasse kann immer die stelle zwischen Konjunktor und erstem Element im Hauptsatz einnehmen: gerade, sogar U.a

\section{KOPULAPART IKELN}

Es handelt sich um die "nur prädikativen Adjektive" der traditionellen Grammatik, also quitt, leid, angst usw.

\section{MODALWORT}

Es handelt sich um partikeln, die die erste stelle im satz einnehmen können und jederzeit als Antwort auf Ja-Nein-Fragen fungieren können: allerdings, freilich, vielleicht u.a.

\section{RANGIERPARTIKELN}

Es handelt sich um Partikeln, die jederzeit die erste stelle im Hauptsatz einnehmen können, aber nicht als Antworten auf Fragen fungieren können: auBerdem, erstens, übrigens u.a.

\section{SATZ ÄQU IVALENTE}

Es handelt sich um partikeln, die nicht im satzzusammenhang vorkommen, sondern, häufig als Antworten, die stelle vollständiger sätze einnehmen (ja, nein, doch usw.).

\section{SUBJUNKTOR}

Es handelt sich um nebensatzeinleitende partikeln (die "unterordnenden Konjunktionen" der traditionellen Grammatik) wie das, obwohl, wenn u.a. 
VERB

Hierunter fassen wir alle konjugierbaren wörter, auch wenn sie defektive Flexionsparadigmen haben, zusammen. Wir gliedern in HAUPTVERBEN, NEBENVERBEN (AUXILIARVERBEN, MODALVERBEN, MODALITÄTSVERBEN u.a.) und FUNKTIONSVERBEN.

\subsection{Wortgruppen}

Grundsätzlich können zu jeder Wortklasse PHRASEN gebildet werden, deren struktur teilweise durch die Valenz des regierenden Wortes (des "Nukleus") bestimmt wird. Wir sprechen dann von ADJEKTIVALPHRASEN, NOMINALPHRASEN, PRÄPOSITIONALPHRASEN USW.

Die Verbalphrase, sowie sie nur aus verbalen Bestandteilen besteht ( hat gemacht, ist benachrichtigt worden, soll haben kommen wollen usw.), bezeichnen wir als VERBALKOMPLEX.

6.3. Der Satz und seine Bestandteile

SATZTYP :

HAUPTSATZ, NEBENSATZ .

SATZART:

beim Hauptsatz: KONSTATIVSATZ, INTERROGATIVSATZ, IMPERATIVSATZ .

beim Nebensatz: ERGÄNZUNGSSÄTZE, ANGABESÄTZE, ATTRIBUTSÄTZE (s. unten).

SATZMUSTER :

Grundstruktur des Satzes, bestehend aus dem Hauptverb und seinen Ergänzungen.

\section{SATZBAUPLAN :}

Satzmuster, in dem zusätzlich der Unterschied zwischen obligatorischen und fakultativen Angaben berücksichtigt ist. 
SATZGLIEDER :

Sie zerfallen in ERGÄNZUNGEN und ANGABEN.

ERGÄNZUNGEN :

Es handelt sich um subklassenspezifisch dem Verb zugeordnete Elemente:

NOMINATIVERGÄNZUNG ("Subjekt")

AKKUSATIVERGÄNZUNG

GENITIVERGÄNZUNG

DATIVERGÄNZUNG

PRÄPOSITIVERGÄNZUNG

SITUATIVERGÄNZUNG

DIREKTIVERGÄNZUNG

NOMINA LERGÄNZUNG

ADJEKTIVALERGÄNZUNG

VERBATIVERGÄNZUNG

\section{ANGABEN}

Es handelt sich um aspezifisch dem Verb zugeordnete Elemente. wir unterscheiden folgende Großklassen:

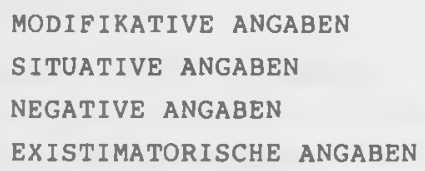

ATTRIBUTE

Sie kommen nur bei wörtern vor, die keine Verben sind; hauptsächlich bei Nomen, Adjektiv, Pronomen.

Je nachdem, ob die Attribute subklassenspezifisch oder aspezifisch ihrem Nukleus zugeordnet sind, unterscheiden wir auch hier Ergänzungen (z.B. NOMENERGÄNZUNGEN) und Angaben ( $\mathrm{z} . \mathrm{B}$. NOMENANGABEN). 


\subsection{Text}

\section{ÄUSSERUNG}

Einzelsprachliche Grundeinheit der Textebene. Die übereinzelsprachliche Texteinheit ist der

\section{SPRECHAKT}

In der Grammatik werden 27 sprechakttypen unterschieden.

\section{TEXTKONNEXION}

Sie wird vor allem sichergestellt durch die Konnektoren.

$\mathrm{Zu}$ ihnen gehören neben den

VERWE ISFORMEN,

hauptsächlich Pronomina, Determinative (in Nominalphrasen)

und Adverbien, die

TEXTORGANISATOREN (z.B. immerhin) und die

GLIEDERUNGSSIGNALE (z.B. nicht wahr?)

Auch die

THEMA-RHEMA-GLIEDERUNG

wirkt nach dem Prinzip der "thematischen Progression" textkonnektiv.

Bei der TEXTWIEDERGABE handelt es sich um formen der sog. direkten und indirekten Rede u.ä. Dieses Textphänomen konnte bisher im Rahmen der satzgrammatik nicht angemessen behandelt werden.

\section{Anmerkungen}

1. Die DVG ist zusammenfassend dargestellt bei Engel 1982.

2 Z.B. in Engel 1970, Seite 27.

3 s. Engel/Mrazovic 1986.

4 Die deutsch-serbokroatische kontrastive Grammatik ist eine Gemeinschaftsarbeit deutscher und jugoslawischer Germanisten. Die wesentlichen Entscheidungen, auch die terminologischer Art, wurden durch Absprache und oft nach langwierigen Diskussionen getroffen. 


\section{Literatur}

\section{Wissenschaftliche Literatur}

Engel, Ulrich: Regeln zur Wortstellung, in: Forschungsbericht des Instituts für deutsche Sprache, Bd. 5, 1970, S.7-148.

Enge1, Ulrich: Syntax der deutschen Gegenwartssprache, Berlin ${ }^{2} 1982$ (1977).

Engel, Ulrich/ Mrazovic, Pavica (Hrsg.): Deutsch-serbokroatische kontrastive Grammatik, exarbeitet von Jovan Djukanovic, Ulrich Engel, Pavica Mrazovic, Hanna popadic, Zoran ziletic. Mit Geleitwort von Rudolf Filipovic, Mïnchen/Novi Sad 1986.

DEUTSCHE LEHRWERKE, IN DEUTSCHLAND (BRD und DDR) hergestellt:

Autofen-Kollektiv: DEuTsCH. Ein Lehrbuch fïr Ausländer, Teil 1, Leipzig 1976 (1959) (unveränderte Neuauflage).

\section{DEUTSCH AKTIV}

Neuner, Gerd et al.: Deutsch aktiv. Ein Lehrwerk für Erwachsene. Lehrbuch 1 , Berlin/Mïchen/wien/zirich 1979.

Neuner, Gerd et al.: Deutsch aktiv. Ein Lehrwerk für Erwachsene. Arbeitsbuch 1, Berlin/München/Wien/Zürich 1983 (1979).

Neuner, Gerd et al.: Deutsch aktiv. Ein Lehrwerk für Erwachsene, Lehrerhandbuch 1. Berlin/Minchen/wien/Ziirich 1983 (1979).

Neuner, Gerd et al.: Deutsch aktiv. Ein Lehrwerk für Erwachsene, Lehrbuch 2 , Berlin/München/wien/Zürich 1982 (1980).

Neuner, Gerd et al.: Deutsch aktiv. Ein Lehrwerk für Erwachsene, Arbeitsbuch 2, Berlin/München/Wien/Zürich 1982 (1980).

Neuner, Gerd et al.: Deutsch aktiv. Ein Lehrwerk für Erwachsene. Lehrerhandbuch 2, Berlin/München/wien/Zürich 1981.

\section{DEUTSCH KONKRET}

Neuner, Gerd et al.: Deutsch konkret. Ein Lehrwerk für Jugendliche. Lehrbuch 1, Berlin/München/Wien/zïrich 1983.

Neuner, Gerd et al.: Deutsch konkret. Arbeitsbuch 1, Berlin/München/wien/ Zürich 1983.

\section{DEUTSCH HIER}

Scherling, Theo et al.: Deutsch hier. Ein Unterrichtswerk für ausländische Arbeitnehmer: Erwachsene und Jugendliche. Lehrbuch, Berlin/München/ Wien/zürich 1982 .

ortmann, Wolf Dieter et al.: Deutsch hier. Ein Unterrichtswerk für ausländische Arbeitnehmer: Erwachsene und Jugendliche. Lehrerhandreichungen, Berlin/München/Wien/zürich 1983. 


\section{DIALOG DEUTSCH}

Becker, Norbert et al.: Dialog Deutsch. Kommunikativer sprachkurs, Arbeitsbuch 1, Carl Duisberg Centren, Köln 1978.

Becker, Norbert et al.: Dialog Deutsch, Kommunikativer Sprachkurs, Textbuch 1, Carl Duisberg Centren, Köln 1978.

\section{DEUTSCH MIT ERFOLG}

Griesbach, Heinz: Deutsch mit Erfolg. Ein Lernprogramm für Erwachsene, Minchen 1983.

\section{GRUNDKURS DEUTSCH}

Schäpers, Roland et al.: Grundkurs Deutsch, Mïnchen 1980.

Schäpers, Roland et al.: Grundkurs Deutsch. Lehrerhandbuch, München 1980.

AUFBAUKURS DEUTSCH

Stalb, Heinrich: Aufbaukurs Deutsch, München 1982.

\section{LERNZIEL DEUTSCH}

Hieber, Wolfgang: Lernziel Deutsch. Deutsch als Fremdsprache, Grundstufe 1 , Minchen 1983.

Gutzat, Bärbel: Lernziel Deutsch, Grundstufe 1, Lehrerhandbuch, Mủnchen 1983.

\section{MITEINANDER}

Bieler, Karl Heinz (Hrsg.): Miteinander. Text- und Arbeitsbücher für Fortgeschrittene in Deutsch als Fremdsprache, Bd. 1-4 (geplant: 6 Bde.), 19791982 .

\section{SPRACHKURS DEUTSCH}

Häussermann, Ulrich et al.: Sprachkurs Deutsch. Unterrichtswerk für Erwachsene. Teil 1 und 2, unter Mitarbeit von Hans-Heinrich wängler, Frankfurt/Wien/Aarau $1978 \mathrm{f}$.

Häussermann, Ulrich et al.: Sprachkurs Deutsch. Unterrichtswerk für Erwachsene, Teil 3 und 4, Frankfurt/Wien/Aarau 1982.

Häussermann, ulrich et al.: Hinweise für Kursleiter (zu Sprachkurs Deutsch), Frankfurt/Wien/Aarau 1979.

\section{THEMEN 1}

Aufderstraße, Hartmut et al.: Themen 1. Kursbuch. Projektbegleitung: HansEberhard Piepho, Minchen 1983.

Eisfeld, Karl-Heinz et al.: Themen 1, Arbeitsbuch Inland. Projektbegleitung: Hans-Eberhard Piepho, Muinchen 1983.

Eisfeld, Karl-Heinz et al.: Themen 1. Arbeitsbuch Ausland, München 1983.

Gerdes, Mechthild et al.: Themen 1, Lehrerhandbuch, München 1984. 
IN POLEN HERGESTELLTE LEHRWERKE:

AKADEMICKI PODRECZNIK JEKYYKA NIEMIECKIEGO

Czochralski, Jan et al.: Akademicki Podrecznik Jezyka Niemieckiego

(Akademisches Lehrbuch der deutschen Sprache), Warszawa 1983.

DER ERSTE SCHRITT

UNSER ALLTAG

ES GIBT IMMER PROBLEME

Grucza, Barbara et al.: Der erste Schritt, Warszawa 1977.

Unser Alltag, Warszawa 1979.

Es gibt imuer Probleme, Warszawa 1979.

(Für die Klassen 1 - 3 der allgemeinbildenden Lyzeen).

LERNT MIT UNS !

Honsza, Joanna; Kozielek, Maria: Lernt mit uns! 4 Bände, Warszawa $1976 \mathrm{f}$.

Honsza, Joanna et al.: Anfang und Fortschritt, Podręcznik dla szkól podstawcych (Lehrbuch für Grundschulen), Wargzawa 1982 .

PRÜFUNGSANFORDERUNGEN :

Baldegger, Markus et al.: KonTAKTSCHWELLE Deutsch als Fremdsprache. Berlin/ Muinchen/wien/zürich 1981.

steger, Hugo (Hrsg. fiur den deutschen Volkshochschul-Verband und das GoetheInstitut): DAS ZERTIFIKAT Deutsch als Fremdsprache, Bonn und München $1985(1972)$. 